\title{
Estimation of the Degree of Crystallinity of Partially Crystalline Polypropylenes Using ${ }^{13} \mathrm{C}$ NMR
}

\begin{abstract}
V. HronskY̌*, M. Koval'aková, P. Vrábel, M. Uhrínová, D. OlČÁK
Department of Physics, Faculty of Electrical Engineering and Informatics, Technical University of Košice, Park Komenského 2, 04200 Košice, Slovakia

The paper deals with a method for the estimation of the degree of crystallinity for partially-crystalline isotacticpolypropylenes (i-PP) using high-resolution solid-state ${ }^{13} \mathrm{C}$ NMR. For this purpose direct polarization ${ }^{13} \mathrm{C}$ MAS NMR spectra were measured for i-PP samples with different degrees of crystallinity at $98^{\circ} \mathrm{C}$. The areas beneath the resonance lines in these spectra correspond to the number of carbons in particular functional groups, while the widths and shapes of the lines reflect the degree of crystallinity, crystalline modifications, distribution of chain conformations and the chain mobility. The ${ }^{13} \mathrm{C}$ MAS NMR spectra, measured using appropriate combination of delay time and high proton decoupling field, made it possible to detect only amorphous domains in the sample. This enabled identification of the lines associated with the amorphous domains in the complete ${ }^{13} \mathrm{C}$ MAS NMR spectra and provided sufficient information for reliable estimation of the degree of crystallinity. The heteronuclear Overhauser enhancement of the ${ }^{13} \mathrm{C}$ NMR signals due to short delay time was taken into account in our calculations.
\end{abstract}

DOI: 10.12693/APhysPolA.126.409

PACS: 61.41.+e, 76.60.-k, 81.05.Lg

\section{Introduction}

The degree of crystallinity is an important morphological characteristic which determines many properties of polymeric materials. It is usually defined in terms of the weight fraction of crystalline domains within a polymer. The evaluation of the degree of crystallinity using ${ }^{13} \mathrm{C}$ NMR is based on the fact that magnetic moments of the nuclei of the same functional group within crystalline and amorphous domains experience different internal magnetic fields and produce different lines in the ${ }^{13} \mathrm{C}$ NMR spectra. The relative number of ${ }^{13} \mathrm{C}$ nuclei within crystalline domains, which is the degree of crystallinity, can be estimated in i-PP samples by the deconvolution of $\mathrm{CH}_{2}$ and $\mathrm{CH}$ lines in quantitative ${ }^{13} \mathrm{C}$ NMR spectra, as was reported in [1] for i-PP with hexagonal structure. The deconvolution of sufficient accuracy of these lines was achieved using two components assigned to ${ }^{13} \mathrm{C}$ nuclei in amorphous and crystalline domains. The ${ }^{13} \mathrm{C}$ NMR spectrum of samples, also containing monoclinic crystals, displays a $\mathrm{CH}_{2}$ line consisting of three overlapping lines. The accuracy of deconvolution could then be lower than for i-PP with hexagonal structure. The results reported in this paper demonstrate that the positions, widths and shapes of lines arising from amorphous domains can be obtained from ${ }^{13} \mathrm{C}$ NMR spectrum recorded with a short delay time between two consecutive scans. These parameters can be then used for deconvolution of the $\mathrm{CH}_{2}$ line in a quantitative ${ }^{13} \mathrm{C}$ NMR spectrum, obtained in an experiment with a long delay time, and then for the calculation of the degree of crystallinity of these samples with comparable accuracy. This procedure was verified

*corresponding author; e-mail: viktor.hronsky@tuke.sk on pelletized $\mathrm{m}-\mathrm{iPP}$ and $\mathrm{ZN}-\mathrm{iPP}$ samples characterized in [1] and the values of degree of crystallinity $54 \%$ and $58 \%$, respectively, are in good agreement with those reported there.

\section{Experimental}

The NMR measurements were carried out on two foil samples (denoted as F1, F2) of i-PP differing in crystallinities, which were supplied by Chemosvit, a.s. Svit (Slovakia). The single-pulse high-resolution solid-state ${ }^{13} \mathrm{C}$ NMR spectra were recorded on $400 \mathrm{MHz}$ Varian solid-state NMR spectrometer at the resonance frequency of approximately $100 \mathrm{MHz}$ and MAS frequency of $10 \mathrm{kHz}$. The ${ }^{13} \mathrm{C}$ MAS NMR measurements were performed at $30^{\circ} \mathrm{C}$ with delay time and high power proton dipolar decoupling (DD) field of $240 \mathrm{~s}$ and $75 \mathrm{kHz}$, respectively, and at $98^{\circ} \mathrm{C}$, with delay times of 1 and $240 \mathrm{~s}$ and DD field of 45 and $75 \mathrm{kHz}$, respectively. Other experimental details and details on DSC experiments can be found elsewhere [1].

\section{Results and discussion}

In general ${ }^{13} \mathrm{C}$ NMR spectra measured for partiallycrystalline i-PP at room temperature consists of three lines at approximately 44,27 and $22 \mathrm{ppm}$ associated with ${ }^{13} \mathrm{C}$ nuclei in the $\mathrm{CH}_{2}, \mathrm{CH}$ and $\mathrm{CH}_{3}$ groups, respectively. If the spectra are recorded using the direct polarization technique, and sufficiently long delay time between two consecutive scans, (in the case of our i-PP samples it is $240 \mathrm{~s}$ ), then the line areas correspond to the number of carbons in the respective groups. The ${ }^{13} \mathrm{C}$ NMR spectra of F2 sample measured at room temperature displays the splitting of $\mathrm{CH}_{3}$ line (Fig. 1A) from which the presence of monoclinic crystals in i-PP foils can be inferred [2]. Due to poor resolution, these spectra do not make it possible to estimate the percentage of crystalline domains in the studied sample. The ${ }^{13} \mathrm{C}$ 
NMR spectra measured at higher temperature $\left(98^{\circ} \mathrm{C}\right)$ give more favourable conditions for evaluations of the degree of crystallinity, since lines associated with $\mathrm{CH}_{2}$ and $\mathrm{CH}$ groups split into two lines at chemical shifts of $44.5 \mathrm{ppm}, 46.4 \mathrm{ppm}$ and $26.6 \mathrm{ppm}, 28.7 \mathrm{ppm}$ produced by carbons within the crystalline and amorphous domains, respectively, and a shoulder observed on the right side of the $\mathrm{CH}_{2}$ line (Fig. 1B).

The partial overlapping of lines associated with the $\mathrm{CH}$ groups, with those associated with $\mathrm{CH}_{3}$ groups, can influence the accuracy of deconvolution, and for this reason the line assigned to $\mathrm{CH}_{2}$ groups (well separated from the neighbouring $\mathrm{CH}$ lines) provides better conditions for estimation of the degree of crystallinity. Since this resonance (Fig. 1B) consists of three strongly overlapping lines, estimation of the degree of crystallinity of this sample requires identification of the lines related either to crystalline or amorphous domains and finding their positions.

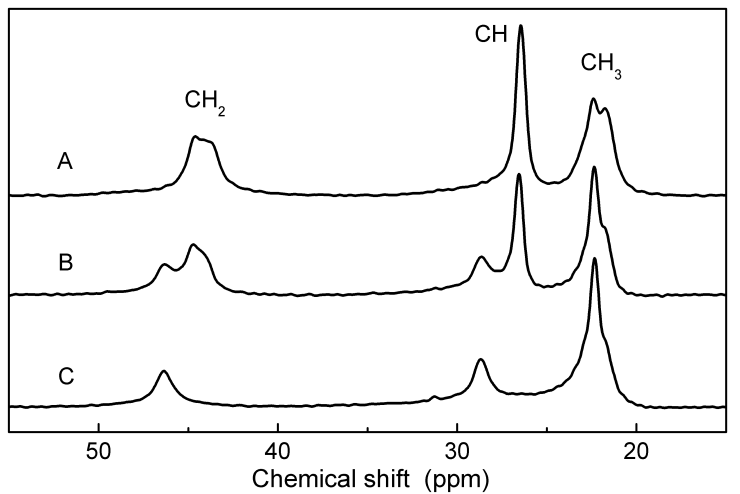

Fig. 1. The ${ }^{13} \mathrm{C}$ MAS NMR spectra for F2 sample measured at MAS rate of $10 \mathrm{kHz}$ and with: delay time of $240 \mathrm{~s}$, DD field of $75 \mathrm{kHz}$ at $30{ }^{\circ} \mathrm{C}$ (A), delay time of $240 \mathrm{~s}$, DD field of $75 \mathrm{kHz}$ at $98^{\circ} \mathrm{C}(\mathrm{B})$, delay time of $1 \mathrm{~s}$, DD field of $45 \mathrm{kHz}$ at $98^{\circ} \mathrm{C}(\mathrm{C})$.

The chemical shifts of the lines related to amorphous domains in i-PP samples can be obtained in a direct polarization experiment with short delay time, since in this case the spin system in crystalline domains due to long spin-lattice relaxation time does not have sufficient time for relaxation. Since the $T_{1}$, measured by the inversion recovery technique, gives the value of $0.2 \mathrm{~s}$ for the amorphous domains of i-PP samples, the ${ }^{13} \mathrm{C}$ NMR spectra measured with the delay time of $1 \mathrm{~s}$ display only signals from amorphous domains in the samples. The ${ }^{13} \mathrm{C}$ NMR spectra of both samples measured at $98^{\circ} \mathrm{C}$ with delay time of $1 \mathrm{~s}$ (Fig. 1C, for F2) made it possible to determine the positions and shapes of the lines related to the amorphous domains and to use these parameters for the deconvolution of the spectra measured with delay times of $240 \mathrm{~s}$.

Due to the fact that heteronuclear Overhauser enhancement of the ${ }^{13} \mathrm{C}$ NMR signals in the spectra measured with the short delay time was observed [3], the am- plitude of these signals cannot be used in the deconvolution of the ${ }^{13} \mathrm{C}$ NMR spectra measured with the long delay time, but the shape, width and position of the amorphous lines were used in the deconvolutions. The results of the calculations of degrees of crystallinity are summarized in the Table. The values obtained from NMR and DSC experiments differ more than those obtained for iPP samples with hexagonal structure [1], which could result from the fact that the structure of foils studied was not pure monoclinic (the ratio of the components in $\mathrm{CH}_{2}$ and $\mathrm{CH}_{3}$ lines in Fig. $1 \mathrm{~A}$ is $1.6: 1$ which differs from theoretically expected $2: 1$ ). This introduces an error in the calculation of the degree of crystallinity from DSC experiments since the value of heat of fusion for $100 \%$ crystalline polypropylene could be lower than that which was adopted for calculations (209 J/g [4]), due to the presence of other i-PP isomorphs.

TABLE

Degrees of crystallinity for i-PP samples, determined from the deconvolution of $\mathrm{CH}_{2}$ lines in ${ }^{13} \mathrm{C} \mathrm{NMR}$ spectra, measured at delay time of $240 \mathrm{~s}$ and from DSC experiments.

\begin{tabular}{c|c|c}
\hline \hline \multirow{2}{*}{ Sample } & \multicolumn{2}{|c}{ Crystallinity (\%) } \\
\cline { 2 - 3 } & NMR $^{*}$ & DSC $^{* *}$ \\
\hline F1 & 63 & 48 \\
\hline F2 & 68 & 52 \\
\hline
\end{tabular}

*relative error estimated from repeated deconvolutions of \pm 4 ,

${ }^{* *}$ relative error of \pm 10 .

\section{Conclusions}

${ }^{13} \mathrm{C}$ NMR experiments performed with short delay time make it possible to determine the shape, width and position of the lines associated with $\mathrm{CH}_{3}, \mathrm{CH}_{2}$ and $\mathrm{CH}$ groups in amorphous domains of i-PP samples. These parameters can be then used for the deconvolution of well separated $\mathrm{CH}_{2}$ line in quantitative ${ }^{13} \mathrm{C}$ NMR spectra and estimation of the degree of crystallinity of i-PP samples with predominant monoclinic structure.

\section{Acknowledgments}

This work was supported by the Slovak Ministry of Education under VEGA project 1/0492/13.

\section{References}

[1] O. Fričová, M. Uhrínová, V. Hronský, M. Kovaĺaková, D. Olčák, I. Chodák, J. Spěváček, eXPRESS Polym. Lett. 6, 204 (2012).

[2] R. Kitamaru, Advances in Polymer Science 137, 41 (1998).

[3] M.H. Levitt, Spin Dynamics: Basics of Nuclear Magnetic Resonance, 2nd ed., Willey, p. 566.

[4] X. Zhang, Y. Zhao, Z. Wang, C. Zheng, X. Dong, Z. Su, P. Sun, D. Wang, C.C. Han, D. Xu, Polymer 46, 5956 (2005). 